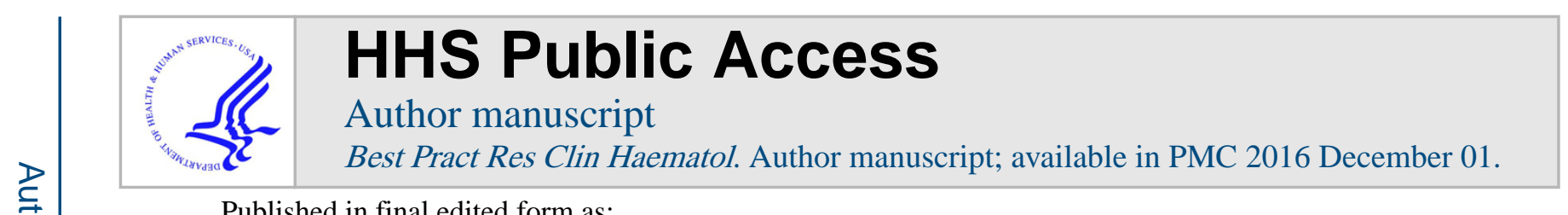

Published in final edited form as:

Best Pract Res Clin Haematol. 2015 December ; 28(4): 269-272. doi:10.1016/j.beha.2015.10.015.

\title{
Hairy cell leukemia: Past, present and future
}

\author{
Bartlomiej M. Getta, MBBS ${ }^{1}$, Jae H. Park, MD², and Martin S. Tallman, MD ${ }^{3,{ }^{*}}$ \\ Bartlomiej M. Getta: gettab@mskcc.org; Jae H. Park: parkj6@mskcc.org \\ ${ }^{1}$ Leukemia Service, Department of Medicine, Memorial Sloan-Kettering Cancer Center, 1275 York \\ Avenue, New York, 10065, USA \\ ${ }^{2}$ Leukemia Service, Department of Medicine, Memorial Sloan-Kettering Cancer Center, 1275 York \\ Avenue, New York, 10065, USA and Weill Cornell Medical College, 1305 York Avenue, New York, \\ 10021, USA \\ ${ }^{3}$ Leukemia Service, Department of Medicine, Memorial Sloan-Kettering Cancer Center, 1275 York \\ Avenue, New York, 10065, USA and Weill Cornell Medical College, 1305 York Avenue, New York, \\ 10021, USA
}

\begin{abstract}
This brief review highlights the sequence of therapeutic milestones and advances in our understanding of the biology of hairy cell leukemia (HCL) with a focus on recent molecular findings and how these may be applied to improve disease outcomes in the future. Targeted therapy is discussed in the context of the recently identified BRAF mutation and other genetic findings.
\end{abstract}

\section{Keywords}

Hairy cell leukemia; BRAF; vemurafenib

\begin{abstract}
Hairy cell leukemia (HCL) is an extremely rare form of leukemia with only 1000 new cases diagnosed in the United States every year [1]. Following its description by Bouroncle in 1958 [2] it was apparent that patients, typically middle-aged Caucasian men, presented with distinct clinical and pathological features including splenomegaly without lymphadenopathy, lymphocytosis and monocytopenia. The morphologic findings are characterized by small lymphocytes bearing fine hair- like cytoplasmic projections with extensive infiltration of reticuloendothelial organs. Advances in treatment have closely followed the discovery of pathogenic mechanisms, including the identification of lower levels of adenosine deaminase in hairy cells relative to normal cells. This has become the target of deoxycoformycin, more commonly known now as pentostatin, enabling proportionally greater inhibition of the
\end{abstract}

\footnotetext{
*Corresponding Author: Martin S. Tallman, TallmanM@mskcc.org.

Conflict of interest

None.

Publisher's Disclaimer: This is a PDF file of an unedited manuscript that has been accepted for publication. As a service to our customers we are providing this early version of the manuscript. The manuscript will undergo copyediting, typesetting, and review of the resulting proof before it is published in its final citable form. Please note that during the production process errors may be discovered which could affect the content, and all legal disclaimers that apply to the journal pertain.
} 
adenosine deaminase in the leukemic cells. As with many rare malignancies, which have idiosyncratic clinical and pathologic features, novel genetic sequencing tools have identified a single, recurrent somatic mutation activating a protein kinase to be present in the overwhelming majority of patients [3].Activating mutations in the BRAF kinase domain at V600 promote hairy cell survival, and characterize the classical form of HCL. This has led to the application of inhibitors targeting BRAF, previously described in patients with melanoma [4].

HCL remains an incurable disease characterized by long periods of remission with eventual relapse and need for re-treatment in many patients. Therapies used in the early 1980s including splenectomy and interferon [5] were replaced with purine analogues (PA) pentostatin and cladribine [6] prior to 1990. Cladribine and pentostatin remain today the accepted first line regimens, with overall response rates (ORR) of $90-100 \%$ and complete responses (CR) of 80-95\% defined as the normalization of blood counts and absence of morphologic evidence of HCL in peripheral blood and bone marrow. Remission is durable with a median progression free survival (PFS) of approximately 9-11 years [7, 8].

The concept of minimal residual disease (MRD) was first applied to HCL in the 1990s. MRD was initially quantified using immunohistochemistry markers, and was identified in $13-26 \%$ of patients following treatment with pentostatin or cladribine [9]. More sensitive tests including flow cytometry and PCR for immunoglobulin gene rearrangement using consensus or sequence specific primers demonstrate MRD in 27-50\% of patients who are in CR $[10,11]$. Application of digital PCR found that all patients treated with vemurafenib had detectable MRD by quantification of mutant BRAF in bone marrow despite being in CR [12]. Patients with detectable MRD have been shown in most studies to have a shorter period of remission but no study has shown that clearance of MRD leads to improvement in survival [13].

Ravandi treated patients with rituximab following initial therapy with cladribine and detected MRD 3 months later using flow cytometry and PCR in 21\% and 30\% of patients respectively. Despite this all patients achieved CR. A prospective trial to investigate whether clearance of MRD using rituximab improves PFS or OS is ongoing. The use of MRD in clinical practice has not been universal; however, it may be important in patients who are likely to have poor responses to upfront PA such as those expressing the immunoglobulin heavy chain variable region (IGHV) 4-34 or TP53 mutations [14]. These molecular factors are not part of routine disease assessment; however, clinical outcomes could potentially be improved by identifying these patients who may benefit from adding secondary agents like rituximab to up- front PA therapy.

Approximately $40 \%$ of patients relapse after 9 years of follow-up. The treatment of relapsed disease is determined by the duration of first remission, with most commentators recommending re-treatment with the same purine analogue used initially if first remission duration was over 2 years [15]. $68 \%$ achieve a second CR while only $47 \%$ achieve a third, highlighting that treatment efficacy is reduced and shorter remissions occur with subsequent treatments [16]. Rituximab is usually combined with purine analogues to treat relapse and improves the CR to $89 \%$ and prolongs the duration of second remission [16]. 
Treatment of purine analogue refractory disease was revolutionized in the last decade with three new classes of therapeutic agents. Immunoconjugates combine the Pseudomonas exotoxin (PE) to the Fv fragment of antibody targeting CD22. These include BL22 and a modified version with higher CD22 affinity called Moxetumomab pasudotox (HA22). These have been studied in phase 1 and 2 trials in PA refractory patients showing ORR of $72 \%$ and $88 \%$ and CR of $47 \%$ and 57\% for BL22 [17] and HA22 [18] respectively. These agents induced a microangiopathic hemolytic disorder in $4-8 \%$ of patients that appears to be their major toxicity. There has been less interest in a CD25-PE conjugate (LMB-2) due to the variability in expression of this antigen [19].

The B cell receptor pathway has been shown to play a role in promoting hairy cell survival in vitro [20]. Recent results of Ibrutinib in 13 patients with HCL were presented in abstract form [21] with an ORR of $46 \%$ and a single CR in relapsed refractory patients, this agent is likely to have less influence in HCL than it has in CLL.

More recent interest relating to HCL has been centered around the anticipated publication of results from a phase 2 trial of vemurafenib, a potent BRAF inhibitor. Vemurafenib is able to reduce ERK phosphorylation and subsequently BRAF-MEK-ERK pathway transcription output, revert hairy cell gene expression to normal and ultimately induce hairy cell apoptosis [22]. Although reports of its efficacy in targeting the major driver mutation in HCL have been published previously [23] the results of a pivotal phase 2 study showing its remarkable clinical efficacy have only recently been presented by a combined effort from Italian and US investigators [12]. Combining the outcomes of the two populations showed that patients with relapsed and/or refractory HCL following prior cladrabine therapy who were treated with a limited course of vemurafenib had an ORR of $98 \%$ and CR 38\%. The RFS was 9 months after a median follow up of 23 months in the Italian cohort while the PFS after 1 year in the US cohort was $73 \%$. Vemurafenib had manageable toxicities with rash, photosensitivity and arthritis the most common and all were reversible with dose reduction or cessation of treatment. The agent was applied for a limited duration because of previous reports suggesting an association with an increased rate of skin cancer [4]. In the HCL cohort 7 out of 50 patients (14\%) developed skin cancers, 1 of which was a melanoma, however all cases were successfully excised, diminishing apprehensions somewhat.

Recent developments have improved our understanding of the genetic and immunological mechanisms of this leukemia. Investigators identified the cell of origin to be a hematopoietic stem cell (HSC) rather than a common lymphoid progenitor by way of identifying the BRAF V600E mutation in HSC. This provides a further reason why targeting mutant BRAF may eradicate the leukemia- initiating cell rather than only the mature clonal lymphocytes, which are the major target of more established therapies. While the BRAF V600E mutation is present in the overwhelming majority of patients with classical HCL, a small percentage of patients lack this mutation but have activation of BRAF kinase due to mutations at exon 11 [24] or at other unrelated loci. The BRAF V600E mutation is absent in Variant HCL (HCLv), which typically lacks expression of CD25. A unique immunologic phenotype lacking the V600E BRAF mutation was found in some cases of variant and classical HCL. This subtype expresses IGHV 4-34 rearrangement [25]. 40\% of patients with HCLv and $10 \%$ with classical HCL were found to have this genotype, which independently conferred a 
shorter OS and PFS following treatment with cladribine [26]. The mechanism behind this is unclear. In total, 50\% of patients of HCLv and IGHV 4-34 expressing classical HCL were found to have an activating mutation in MEK1 that is also involved in signaling down to MAP-kinase pathway. Other mutations identified in BRAF wild type patients occurred in TP53, U2AF1 and ARID1A, which have all been described in B-cell malignancies [27] and in epigenetic modifiers EZH2 and KDM6AM [28]. A recent report identified CDKN1B to be the second most commonly mutated gene in HCL occurring in $16 \%$ of patients all of whom also had the BRAF V600E substitution. This mutation has been described in solid organ malignancies and did not impact survival or cladrabine response in the population analyzed [28].

Future directions in management are likely to focus on optimizing therapy of relapsed patients. Whilst the ORR to vemurafenib approached $100 \%$, most patients relapse within months of discontinuing therapy thus ongoing treatment may be necessary. Mutations in BRAF and genes in convergent pathways have been identified in patients refractory to vemurafenib [12]. Clones baring these mutations may be selected out due to the intermittent drug dosing used in the identified trials. Addition of trametinib, a MEK inhibitor [22] to vemurafenib or dabrafenib may bypass the mutations acquired upstream to MEK. Trametinib has been shown to elicit stronger ERK dephosphorylation and induces more hairy cell apoptosis than either BRAF inhibitor alone. Given the identification of MEK1 mutations in some BRAF wild type IGHV 4-34 HCL, which includes patients with HCLv, drugs targeting this pathway may also be explored in this disease subgroup. Finally, investigators are likely to further explore the role of the bone marrow microenvironment in dampening the effects of BRAF inhibition [22] and the critical role played by the spleen in clearing hairy cells [12].

\section{References}

1. Morton LM, et al. Lymphoma incidence patterns by WHO subtype in the United States, 1992-2001. Blood. 2006; 107(1):265-276. [PubMed: 16150940]

2. Bouroncle BA, Wiseman BK, Doan CA. Leukemic reticuloendotheliosis. Blood. 1958; 13(7):609_ 30. [PubMed: 13560561]

3. Tiacci E, et al. BRAF mutations in hairy-cell leukemia. N Engl J Med. 2011; 364(24):2305-15. [PubMed: 21663470]

4. Chapman PB, et al. Improved survival with vemurafenib in melanoma with BRAF V600E mutation. N Engl J Med. 2011; 364(26):2507-16. [PubMed: 21639808]

5. Quesada JR, et al. Alpha interferon for induction of remission in hairy-cell leukemia. N Engl J Med. 1984; 310(1):15-8. [PubMed: 6689734]

6. Piro LD, et al. Lasting remissions in hairy-cell leukemia induced by a single infusion of 2chlorodeoxyadenosine. N Engl J Med. 1990; 322(16):1117-21. [PubMed: 1969613]

7. Else M, et al. Long-term follow-up of 233 patients with hairy cell leukaemia, treated initially with pentostatin or cladribine, at a median of 16 years from diagnosis. Br J Haematol. 2009; 145(6):73340. [PubMed: 19344416]

8. Goodman GR, et al. Extended follow-up of patients with hairy cell leukemia after treatment with cladribine. J Clin Oncol. 2003; 21(5):891-6. [PubMed: 12610190]

9. Tallman MS, et al. Minimal residual disease in patients with hairy cell leukemia in complete remission treated with 2-chlorodeoxyadenosine or 2-deoxycoformycin and prediction of early relapse. Clin Cancer Res. 1999; 5(7):1665-70. [PubMed: 10430066] 
10. Lopez Rubio M, et al. Hairy cell leukemia treated initially with purine analogs: a retrospective study of 107 patients from the Spanish Cooperative Group on Chronic Lymphocytic Leukemia (GELLC). Leuk Lymphoma. 2014; 55(5):1007-12. [PubMed: 23885799]

11. Sausville JE, et al. Minimal residual disease detection in hairy cell leukemia. Comparison of flow cytometric immunophenotyping with clonal analysis using consensus primer polymerase chain reaction for the heavy chain gene. Am J Clin Pathol. 2003; 119(2):213-7. [PubMed: 12579991]

12. Tiacci E, et al. Targeting Mutant BRAF in Relapsed or Refractory Hairy-Cell Leukemia. N Engl J Med. 2015; doi: 10.1056/NEJMoa1506583

13. Park JH, Tallman MS. Left behind: should minimal residual disease be treated in hairy cell leukemia? Leuk Lymphoma. 2014; 55(5):971-2. [PubMed: 24512318]

14. Forconi F, et al. Hairy cell leukemias with unmutated IGHV genes define the minor subset refractory to single-agent cladribine and with more aggressive behavior. Blood. 2009; 114(21): 4696-702. [PubMed: 19667403]

15. Robak T, et al. Hairy cell leukaemia: ESMO Clinical Practice Guidelines for diagnosis, treatment and follow-updagger. Ann Oncol. 2015; 26(Suppl 5):v100-v107. [PubMed: 26269205]

16. Else M, et al. Rituximab with pentostatin or cladribine: an effective combination treatment for hairy cell leukemia after disease recurrence. Leuk Lymphoma. 2011; 52(Suppl 2):75-8. [PubMed: 21504288]

17. Kreitman RJ, et al. Phase II trial of recombinant immunotoxin RFB4(dsFv)-PE38 (BL22) in patients with hairy cell leukemia. J Clin Oncol. 2009; 27(18):2983-90. [PubMed: 19414673]

18. Kreitman RJ, et al. Phase I trial of anti-CD22 recombinant immunotoxin moxetumomab pasudotox (CAT-8015 or HA22) in patients with hairy cell leukemia. J Clin Oncol. 2012; 30(15):1822-8. [PubMed: 22355053]

19. Robbins DH, et al. Hairy cell leukemia, a B-cell neoplasm that is particularly sensitive to the cytotoxic effect of anti-Tac(Fv)-PE38 (LMB-2). Clin Cancer Res. 2000; 6(2):693-700. [PubMed: 10690555]

20. Sivina M, et al. The bruton tyrosine kinase inhibitor ibrutinib (PCI-32765) blocks hairy cell leukaemia survival, proliferation and B cell receptor signalling: a new therapeutic approach. $\mathrm{Br} \mathbf{J}$ Haematol. 2014; 166(2):177-88. [PubMed: 24697238]

21. Jones, Jeffrey A., et al. Safety and efficacy of the bruton tyrosine kinase inhibitor Ibrutinib in patients with Hairy Cell Leukemia: Interim resuls of a phase 2 study. Presented at the 20th Congress of the European Haematology Association (EHA); June 14, 2015; Austria, Vienna. Abstract S790http://learningcenter.ehaweb.org/eha/2015/20th/103204/ jeffrey.jones.safety.and.efficacy.of.the.bruton.tyrosine.kinase.inhibitor.html?f=m3

22. Pettirossi V, et al. BRAF inhibitors reverse the unique molecular signature and phenotype of hairy cell leukemia and exert potent antileukemic activity. Blood. 2015; 125(8):1207-16. [PubMed: 25480661]

23. Dietrich S, et al. BRAF inhibition in refractory hairy-cell leukemia. N Engl J Med. 2012; 366(21): 2038-40. [PubMed: 22621641]

24. Tschernitz S, et al. Alternative BRAF mutations in BRAF V600E-negative hairy cell leukaemias. Br J Haematol. 2014; 165(4):529-33. [PubMed: 24433452]

25. Xi L, et al. Both variant and IGHV4-34-expressing hairy cell leukemia lack the BRAF V600E mutation. Blood. 2012; 119(14):3330-2. [PubMed: 22210875]

26. Arons E, Kreitman RJ. Molecular variant of hairy cell leukemia with poor prognosis. Leuk Lymphoma. 2011; 52(Suppl 2):99-102. [PubMed: 21599610]

27. Waterfall JJ, et al. High prevalence of MAP2K1 mutations in variant and IGHV4-34-expressing hairy-cell leukemias. Nat Genet. 2014; 46(1):8-10. [PubMed: 24241536]

28. Dietrich S, et al. Recurrent CDKN1B (p27) mutations in hairy cell leukemia. Blood. 2015; 126(8): 1005-8. [PubMed: 26065650] 


\section{Practice Points and Research Agenda}

- $\quad$ Classical hairy cell leukemia is characterized by the activating mutation of BRAF at V600.

- Vemurafenib is effective in patients with hairy cell leukemia who have relapsed or are refractory to cladribine and have the BRAF V600E mutation.

- $\quad$ Other genetic mutations have been identified in patients with hairy cell leukemia although their implications in disease treatment and outcome have not been defined. 